\title{
El proyecto de aula como estrategia didáctica en el marco de la enseñanza para la comprensión
}

The classroom project as a didactic strategy in the framework of teaching for understanding

Lilia María Barrios Oviedo, limarbao@gmail.com

Institución Educativa Aguas Negras, Colombia

Martha Abigail Chaves Silva,machsi7@gmail.com

Colegio Visión Mundial Montería, Colombia

\section{Resumen}

Este artículo presenta resultados derivados del proyecto de investigación «El proyecto de aula como estrategia didáctica en el marco del modelo pedagógico enseñanza para la comprensión», que se realizó en la Maestría en Educación de la Universidad de Córdoba-SUE-CARIBE. El artículo tiene como propósito mostrar la incidencia de los proyectos de aula y del modelo pedagógico Enseñanza para la Comprensión (EpC) en la calidad educativa, en comunidades en condiciones de pobreza y vulnerabilidad, en el Colegio Visión Mundial de Montería (Córdoba). La metodología se sustenta en el modelo de la investigación-acción con un enfoque cualitativo; asimismo, se encuentra motivada por la comprensión y transformación de los fenómenos sociales y no por la pretensión de comprobar verdades. El modelo pedagógico EpC y los proyectos de aula se han configurado por medio del reconocimiento y reconstrucción de las voces de los sujetos desde su propio contexto educativo y social, entendiendo que estos son capaces de contribuir a su propia formación y desarrollo a partir del autorreconocimiento. De igual manera, se asume como una apuesta de reconstrucción de lo humano en el marco de la complejidad de lo social. Así, la reflexión sobre la participación de la comunidad, mediada por el diálogo sobre el modelo pedagógico y el trabajo por proyectos, se ha venido convirtiendo en un pilar del proyecto educativo institucional (PEI).

Palabras claves: Comunidades vulnerables, enseñanza para la comprensión, enseñanza por proyectos, prácticas pedagógicas, proyectos de aula.

\section{Abstract}

This article presents the results of the research project «The classroom project as a didactic strategy in the framework of the Teaching for Understanding» which was carried out in the M.A of Education at Universidad de Cordoba - SUE-CARIBE. The article aims to show the incidence of the classroom projects and the Teaching for Understanding pedagogical model (TfU) on the quality of education, in communities with poverty and vulnerability conditions at Colegio Visión Mundial in Montería, Cordoba. The methodology of the project is Action research and follows a qualitative paradigm. This is motivated by the comprehension and transformation of the social phenomena, rather than the attempt to look for truths. The model and the classroom projects have been set up through the recognition and reconstruction of the voices of the subjects from their own educational and social context, understanding that these can contribute to their training and development from the self-recognition. In the same way, they are assumed as an intent for reconstruction human in the framework of the complexity of the social. Thus, reflection about community participation, mediated by the dialogue about the pedagogical model and project work, has become a pillar of the institutional educative project (PEI).

Keywords: Vulnerable communities, teaching for understanding, teaching through projects, teaching practices, classroom projects. 


\section{Introducción}

Este estudio se desarrolla en el Colegio Visión Mundial de la Ciudad de Montería (Córdoba), con estudiantes desde el grado preescolar hasta la media, que a menudo están implicados en la primera línea de los conflictos sociales violentos, circunstancia que los subsume en condiciones de alta vulnerabilidad e inequidad, impidiéndoles desarrollar sus potenciales como sujetos sociales. En consideración con lo anterior, se tuvieron en cuenta fundamentos teóricos y conceptuales con respecto a las tendencias pedagógicas europeas, latinoamericanas y colombianas; además de las estrategias pedagógicas y didácticas, en el marco de los proyectos de aula y el modelo pedagógico Enseñanza para la Comprensión (EpC).

El proceso investigativo se asume bajo un enfoque cualitativo-interpretativo, con base en el método de investigación-acción (IA), que involucra a diversos agentes de la comunidad educativa, como lo son los organismos académicos, administrativos y de desarrollo comunitario que acompañan y guían los procesos de enseñanza y aprendizaje, dentro y fuera de la escuela.

Los resultados parciales han permitido evidenciar que es posible el desarrollo social inclusivo a partir de un trabajo reflexivo, colectivo y participativo, con prácticas pedagógicas exitosas y replicables, a partir del desarrollo de proyectos de aula en el marco del modelo pedagógico EpC en comunidades educativas de alta vulnerabilidad.

\section{Problema y contexto}

La educación ha desarrollado en los últimos años un proceso complejo y diverso, debido en parte a las demandas del contexto sociocultural, lo mismo que a los cambios en la economía, la política y las relaciones entre países y regiones, aspectos que hacen de la educación una herramienta necesaria para asegurar la futura viabilidad hacia la sostenibilidad, la sustentabilidad y el desarrollo.

En el caso de América Latina, según el documento Hacia la Educación de Calidad para Todos al 2015 (Unesco, 2013), se analizan grandes avances y diferencias en las estrategias del crecimiento y aceleración de los procesos de desarrollo económico en los estados. En ese sentido, la educación da respuestas a los requerimientos de la transformación productiva y a las demandas de participación, en el marco de la democratización de nuestras sociedades e integración regional. Esta afirmación no deja de reconocer las asimetrías y desigualdades existentes en la dimensión económica del desarrollo.

En Colombia, según el interés del Gobierno Nacional, desde la prospectiva de los planes de desarrollo, este proceso se centra en una educación orientada a intervenir en los niveles de competitividad (propender por un ingreso cada vez más fuerte en la economía del país) y en la calidad de vida de los integrantes de la sociedad (Plan Nacional Decenal de Educación 2006-2015). Sin embargo, la ampliación de la cobertura, el mejoramiento de la calidad, la pertinencia y eficiencia, dados los escasos recursos para la operación directa de la educación y su administración, aún no evidencian los efectos internos de dicha política en las instituciones educativas.

Como la mayoría de los departamentos del Caribe colombiano, el departamento de Córdoba posee ciertas particularidades sociales, económicas, políticas, ambientales y culturales. Evidenciándose desde hace varios años graves problemas de violencia, sobre todo en el área rural. Así, las poblaciones han llegado a ser víctimas del desplazamiento y en su proceso de reubicación deben afrontar desafíos de superación personal en situaciones adversas. Según Negrete y Garcés (2010), estos procesos migratorios han propiciado el aumento de la población en ciudades como Montería (capital de Córdoba), que cuenta con más de cuarenta asentamientos subnormales, todos ellos receptores de una población desplazada de aproximadamente 24.484 inmigrantes, como consecuencia de la violencia política y económica. La situación descrita contribuye a la creación de una sociedad que se debate 
entre la pobreza y la inequidad, aumentando las desigualdades, la desesperanza y el sentimiento de injusticia. El problema ha ido creciendo en la ciudad, tanto así que la población en edad escolar está a menudo en la primera línea de los conflictos violentos, haciendo que los niños crezcan en condiciones de alta vulnerabilidad e inequidad, y por consiguiente, impidiéndoles desarrollar sus potenciales como sujetos sociales.

Lo anterior permite comprender la decisión que tomó la organización de tipo humanitario Visión Mundial en el año 2000, de fundar una institución educativa que compensara las desventajas de los estudiantes más pobres y que mejorase la calidad de los procesos de aprendizaje, aminorando la fuerte estratificación que persiste en el sistema educativo, los índices de bajo rendimiento académico, el trabajo infantil y el analfabetismo. En esta perspectiva de trabajo adquiere sentido el lema del PEI de la institución «Formando en valores por un nuevo ciudadano», dadas las circunstancias de la problemática social, política y económica de la ciudad, la región y lógicamente, de las familias.

En este marco, el modelo pedagógico EpC y la enseñanza por proyectos hacen énfasis en ofrecer una educación para la vida, incentivando el desarrollo de competencias ciudadanas y la participación activa de los padres de familia en el proceso educativo. Esta propuesta es innovadora para el sistema escolar predominante, pues incentiva y articula las pedagogías activas mediante una aproximación crítica al contexto social y valora los presaberes de los estudiantes que viven en condiciones de pobreza y vulnerabilidad. En esta perspectiva, se considera que el rol del profesor incide de manera directa en el desarrollo del pensamiento del alumno y en la adquisición de su aprendizaje, para lo cual es pertinente orientar las prácticas pedagógicas a través de proyectos de aula, desde su planificación hasta su evaluación, entendidos como una estrategia didáctica integral.

Por otro lado, la Institución Educativa Visión Mundial cuenta con un centro interactivo destinado a que niños, adolescentes, jóvenes, adultos y docentes, no solo del colegio sino de otras instituciones educativas de Montería - especialmente aquellas que se encuentran ubicadas en las comunidades más pobres-, sean capacitados en alfabetización digital. Desde esta perspectiva, el empoderamiento de la gestión escolar permite apostarle al aumento de la calidad educativa a partir de la generación de nuevos escenarios, donde el maestro reflexiona sobre su práctica pedagógica y la reinventa desde la perspectiva de una mediación tecnológica (Siemens, 2004). Para ello, como estrategia de impacto social y educativo sobre las familias de la institución, se desarrolla un proceso de formación de docentes en competencias tecnológicas que comprende dos fases: alfabetización digital y herramientas tecnológicas para el aula desde la Web 2.0.

Considerando esta dinámica educativa, la gestión académica, a través de los proyectos de aula, se consolida como estrategia pedagógica que prioriza procesos de enseñanza y aprendizaje innovadores y significativos, facilitando la construcción de identidades colectivas mediante diversas interacciones preconcebidas, finalidades y programaciones de áreas académicas enfocadas en el trabajo por contenidos actitudinales, procedimentales y conceptuales.

En este orden de ideas, según Zabala (1995), un contenido conceptual es un aprendizaje significativo de conceptos asociados a hechos particulares que sirven para comprender e interpretar diversas situaciones. Por otra parte, de acuerdo con Coll y Valls (1993), los contenidos procedimentales son el conjunto de acciones ordenadas orientadas a la consecución de una meta. Asimismo, señala Sayago (2003), los contenidos actitudinales «se refieren a cómo piensan y qué valoran los alumnos y, sobre todo, cuáles actitudes asumen ante situaciones de conflicto que requieran ayuda, demostraciones de afecto, actos de solidaridad, entre otras» (p. 421).

De igual forma, la gestión del currículo debe estar orientada a una comprensión profunda del por qué, para qué, cómo y sobre qué hay que formar; también debe pensar y dar respuesta a los problemas 
del hombre frente a un mundo cada vez más degradado; además debe considerar los conflictos sociales que se padecen a nivel de las comunidades, de las familias y de la escuela misma (Doria, 2011).

En este sentido, la gestión escolar proyecta un plan estratégico para tres años con los siguientes componentes: a)calidadeducativa,b) crecimientodeingresos, penetraciónen elmercado, posicionamiento y diferenciación de marca, c) fidelización del cliente. Estos componentes se encuentran definidos año por año, con objetivos estratégicos, indicadores de impacto, metas de resultado e indicadores de resultado, para así obtener las respectivas evaluaciones y planes de mejoramiento. El mencionado plan estratégico se elabora con el acompañamiento de las gestiones escolares y en concordancia al ideario que se consolida desde el modelo pedagógico $\mathrm{EpC}$ y la enseñanza por proyectos.

De acuerdo con lo descrito, para este artículo académico se estableció la siguiente pregunta que orienta este trabajo investigativo: ¿Cómo contribuyen los proyectos de aula en la dinamización de los procesos de enseñanza y aprendizaje en el marco del modelo pedagógico Enseñanza para la Comprensión?

\section{Marco Teórico}

En la actual sociedad del conocimiento y la innovación, la búsqueda incesante de nuevas formas de concebir y entender el proceso de enseñanza y aprendizaje se convierte en la principal preocupación de los educadores e instituciones, pues deben asumir situaciones cada vez más complejas y dinámicas. Una sociedad heredera de la nanotecnología, la cibernética, las transformaciones sociales, las revoluciones del siglo XX, la Primavera Árabe, el conflicto armado, las luchas propias de las relaciones de poder, la cada vez más delgada línea que divide los Estados, solo por mencionar unos escenarios, implica un hecho por el cual debemos preguntarnos: ¿Cómo debe ser el proceso de enseñanza? ¿Qué elementos influyen en los procesos de enseñanza y aprendizaje de tal manera que los potencien? ¿Cómo crear escenarios educativos que permitan afrontar las particularidades de los estudiantes? ¿Cómo podemos crear estrategias educativas que posibiliten transformaciones educativas en contextos diversos y complejos a partir de los fundamentos teóricos contemporáneos? ¿Cómo podemos obtener recursos de aprendizaje que permitan enriquecer nuestro quehacer pedagógico a partir de experiencias educativas internacionales? A partir de los anteriores interrogantes se aborda una revisión de algunos aportes teóricos que dan luces a este artículo investigativo.

\section{El modelo pedagógico Enseñanza para la Comprensión como oportunidad de cambio y transformación}

En este apartado se identifican los fundamentos conceptuales y teóricos del modelo pedagógico Enseñanza para la Comprensión, entendido como ruta potenciadora del aprendizaje a partir del uso flexible y consensuado de los conocimientos previos. Se abordan las teorías del Modelo Enseñanza para la Comprensión a partir de las visiones de autores como Perkins (1999), Pogré (2001), Vázquez (2011) y Wiske (1999), entre otros investigadores que proponen experiencias educativas que ponen al descubierto particularidades de la enseñanza para la comprensión, tales como la participación activa del alumnado, el papel de la investigación, el error como fuente de aprendizaje, el trabajo cooperativo, el uso de materiales y recursos diversos y la evaluación continua y participativa.

Se asume el modelo pedagógico EpC y los proyectos de aula, como propuesta metodológica cuyo propósito pedagógico fundamental es desarrollar sujetos capaces de pensar por sí mismos y de actuar de manera responsable, comprometidos en asumir una actitud investigativa y crítica, reconociéndose como protagonistas del aprendizaje mediante el diálogo de saberes y experiencias previas. De esta forma, se provoca que los estudiantes activen los esquemas y preconcepciones de su 
El proyecto de aula como estrategia didáctica

estructura semántica experiencial, para reafirmarlos o reconstruirlos, a la luz del potencial cognitivo que representan los nuevos conceptos de la cultura y los conocimientos públicos con los que ahora se ponen en contacto para resolver los problemas de su vida cotidiana (Gimeno \& Pérez, 1996).

\section{La escuela como institución potenciadora del aprendizaje}

En este punto se analiza la importancia de los sistemas educativos en la consolidación de una organización y cultura institucional de aprendizajes comprensivos; así mismo se reflexiona sobre el verdadero papel de las instituciones educativas, las cuales deben estar preparadas para grandes retos en materia de inclusión y pertinencia.

No podemos permanecer de espaldas a las especificidades y necesidades de estudiantes que exigen cada vez más atención a sus realidades educativas. Hacer una apuesta por la promoción de una cultura de pensamiento, es el objetivo al que le debe apuntar toda institución.

Necesitamos de una generación de estudiantes proactivos, con mentalidad ganadora, que se involucren en el proceso de aprendizaje, que puedan controlar los flujos indiscriminados de información, capaces de aislar los factores irrelevantes y de utilizar el conocimiento para solucionar los problemas de manera creativa e innovadora. Por otra parte, en este proceso de construcción del sujeto, el estudiante y el maestro se reconocen en un espacio formativo dialogante y compartido donde se toma conciencia de las insuficiencias de los esquemas mentales habituales y se valoran las potenciales formas de analizar y transformar la realidad social (Barrios y Chaves, 2014).

El desarrollo de esta propuesta pedagógica, permite la consolidación de sujetos activos, que exponen, preguntan, dialogan y actúan con el saber. Según Not (1992), es un proceso dialogante que forma individuos autónomos que toman decisiones sobre su proceso, que cuentan con mayores grados de autoconciencia en torno a sus procesos y que cualifica la planeación de sus actividades.

Lo anterior invita a reflexionar en torno al compromiso que deben asumir los docentes para la creación de ambientes de aprendizaje mediante la aplicación de situaciones didácticas diversas en donde el estudiante se reconozca desde sus particularidades y necesidades.

\section{Los proyectos de aula como estrategia didáctica integradora}

El aula se considera como el espacio escolar creado para construir conocimiento y establecer procesos de enseñanza y aprendizaje, es un lugar de convivencia e intercambio educativo entre los estudiantes y el docente. En la antigüedad, se consideraba como un palacio del príncipe soberano, en educación, un lugar de respeto y de reflexión (Rivas y Revelo, 2007).

Es en el aula donde el docente y sus estudiantes generan un contrato pedagógico que cumplen ambas partes en pos de construir experiencias en grupo, respetando las condiciones, compartiendo sentimientos de solidaridad y colaboración; es un lugar que se convierte en laboratorio o taller; en algunas ocasiones estos espacios son móviles y su entorno transforma el ambiente (Rivas y Revelo, 2007). Según Guzmán, Graciela y Jiménez (1991):

En lo general, se reconoce el aula como espacio de producción y reproducción de contenidos ideológicos, culturales, relaciones sociales que lo crean y lo mantienen. En este sentido, se puede pensar el aula como un espacio donde se juega un orden social y cultural, así como diversas manifestaciones de su oposición (p. 338).

«Desde esta perspectiva, el conocimiento se construye de manera activa por quien aprende a través de la interacción con otros en un medio social, y el aprendiz puede elaborar personalmente lo 
aprendido, logrando con ello que el aprendizaje le sea funcional y significativo» (Denegri, 1996, citado en Denegri y Martínez, 2003, p. 3). Ello implica que el alumno se enfrenta a los nuevos contenidos con una serie de conceptos, representaciones y conocimientos ya adquiridos y teorías propias que le permiten explicarse el mundo.

En este sentido, en el proceso de enseñanza-aprendizaje los proyectos de aula son prácticas fundamentalmente interactivas en las que resultan tan importantes las relaciones maestro-alumno como aquellas que establecen los alumnos entre sí. Por ello, los proyectos de aula favorecen el intercambio de información, la confrontación de puntos de vista distintos, y en esta actividad, el profesor debe estar especialmente atento a las interacciones que se producen en el grupo, interviniendo para propiciar que se analicen y resuelvan los conflictos en un clima de aceptación, ayuda mutua, cooperación y tolerancia. Ello implica favorecer la autonomía de los escolares en la toma de decisiones, asumiendo sus responsabilidades como miembros de un grupo. Desde esta mirada, el aprendizaje significativo cobra gran importancia, y el proyecto se establece como una alternativa novedosa donde el estudiante genera la construcción de conocimiento en una amplia gama de situaciones y circunstancias (Coll, 1981).

\section{Método}

«El saber pedagógico se construye a través de la reflexión en la acción o conversación reflexiva con la situación problemática». (Schön, 1987, p. 49). Esta investigación es de tipo cualitativo, sustentada en el método investigación-acción, ya que está motivada por búsquedas y no por la pretensión de comprobar verdades; Tampoco le asiste la certidumbre sino la inquietud de construir una significación acerca de una realidad, posibilitando a los participantes actuar de manera reflexiva y colectiva sobre los problemas que los aquejan, donde los procesos educativos tienen como función trascendental la transformación humana.

El enfoque cualitativo, como actividad sistémica orientada a la comprensión en profundidad de fenómenos educativos y sociales (Sandín, 2003), permite indagar, profundizar y describir, en este caso, ¿cuál es el impacto educativo del modelo Enseñanza para la Comprensión y los proyectos de aula en la comunidad visionaria? En este sentido, Lawrence Stenhouse (1984), trabajó intensamente en este enfoque para la innovación e investigación-acción, basándose en la propuesta de Lewin (1948), en escenarios propiamente humanísticos, que es la perspectiva asumida en esta investigación, a partir de prácticas educativas exitosas.

En este orden de ideas, se definieron unas fases de investigación con sus correspondientes técnicas e instrumentos, atendiendo los planteamientos de Le Compte, Millroy \& Preissle (1995), quienes señalan que la investigación cualitativa es aquella que extrae descripciones a partir de observaciones que adoptan las formas de entrevistas, grupos de discusión, narraciones, diarios de campo, transcripciones de audio y video, y registros escritos de todo tipo. La investigación-acción propuesta en este estudio, referido al modelo pedagógico EpC y los proyectos de aula, se propuso como un modelo cíclico de reflexión-acción-reflexión en el que se reorganiza la relación entre conocer y hacer, entre sujeto y objeto, configurando y consolidando con cada paso la capacidad de autogestión de los implicados. Así, se establece como un contexto investigativo más abierto y procesual (Bru \& Basagoiti, 2002).

A partir de lo anterior, se plantearon tres fases interrelacionadas: problema, planeación, ejecución y reflexión, entendida esta última como una evaluación continua. En este sentido, las fases de la investigación implican un diagnóstico, la construcción de planes de acción y la ejecución de dichos planes. Asimismo, la reflexión, evaluación y mejoramiento continuo permiten a los involucrados en la investigación redimensionar, reorientar o replantear nuevas acciones, en atención a las reflexiones 
realizadas y la oportunidad de construir conocimiento.

Por su parte, Kemmis (1993), las incluye en cuatro momentos interrelacionados e identificados como: planificación, acción, observación y reflexión. Mientras que, para Pérez Serrano (1998), son pasos o etapas para el acercamiento con la metodología investigación-acción, que se inician con el diagnóstico de una preocupación temática o problema; luego vienen la construcción del plan de acción, la puesta en práctica del referido plan y su respectiva observación, la reflexión e interpretación de resultados y la replanificación, si fuera necesaria.

En la Figura 1 se representan las fases de la investigación, las cuales están permeadas por una dinámica de reflexión y evaluación continua en la que los actores dinamizan cada fase para avanzar en las comprensiones y las acciones mismas hacia niveles cada vez más avanzados, produciendo conocimiento.

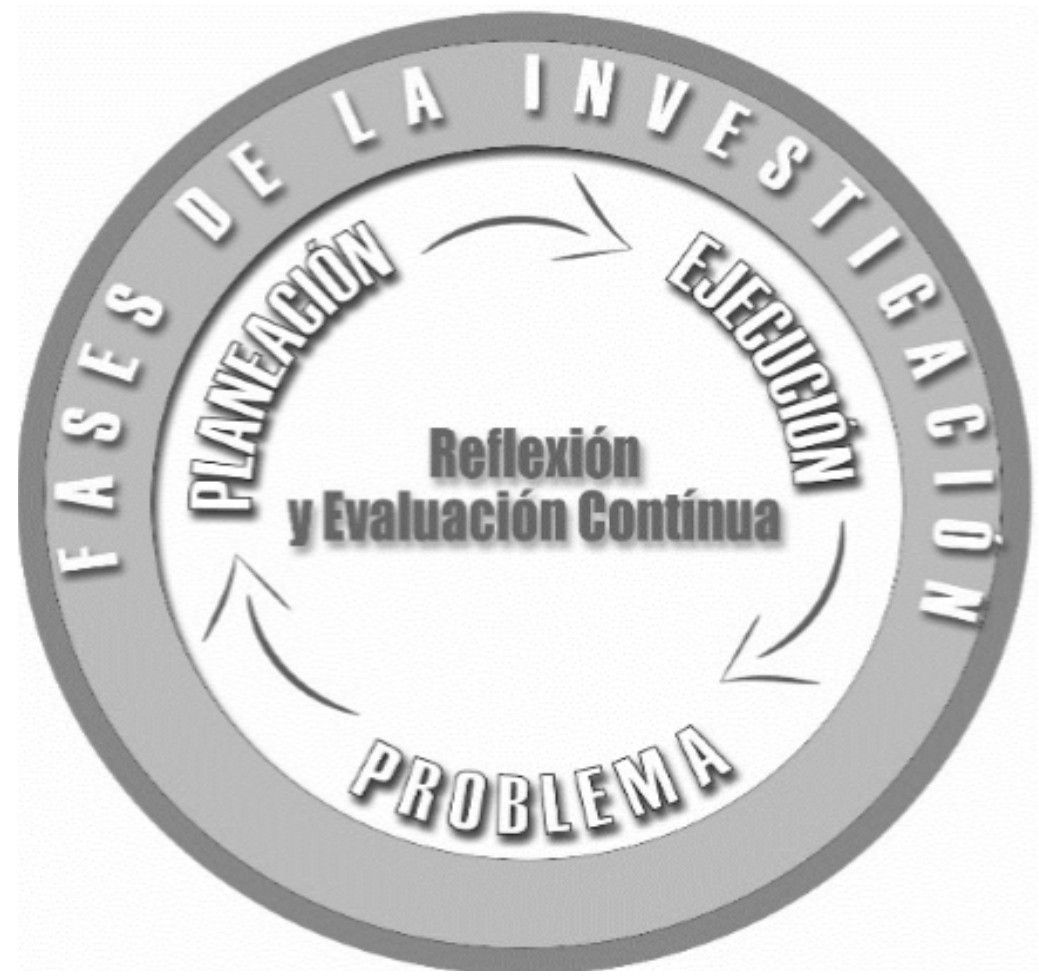

Figura 1. Modelo del método de investigación-acción asumido sobre la experiencia. Fuente: Chaves y Barrios, 2015.

En este proyecto, la investigación-acción busca la reflexión y evaluación continua para el logro de los objetivos, las estrategias y las transformaciones de las gestiones institucionales y educativas; En otras palabras, es una forma de búsqueda autorreflexiva para comprender y perfeccionar las prácticas educativas en las interacciones entre pares, estudiantes, los diferentes miembros de la comunidad educativa y el contexto.

La idea expuesta precisa que la reflexión y evaluación continua en las fases de esta investigación son un proceso cíclico donde el docente, mediante la evaluación continua, busca el cumplimiento y logro de los objetivos y la transformación de las estrategias pedagógicas de manera planeada y organizada, en torno a la construcción de nuevos conocimientos y saberes pedagógicos, haciéndolos pertinentes a las necesidades educativas del medio.

La reflexión y evaluación continua posibilitan la sistematización de experiencias y reflexiones de las gestiones institucionales y educativas con capacidad de producción académica, generando 
cambios en las prácticas pedagógicas y formativas que se están requiriendo y apunta hacia la calidad educativa, de tal manera que esta sea asumida como un centro de investigación y desarrollo de las diferentes formas de la subjetividad humana (Rodríguez, 2000).

Por otra parte, a partir del colectivo de 50 docentes, administrativos y directivos que laboran en el establecimiento educativo Visión Mundial, se estableció y consolidó La Comisión de Aprendizaje y Evaluación Continua (La CAEC), conformada por 8 integrantes. La comisión se asume como un proceso cíclico de la investigación sobre la gestión institucional, problemas individuales y colectivos, la planeación y práctica pedagógica del maestro, la reflexión, evaluación y mejoramiento continuo, estableciéndose los correspondientes parámetros para contactar otros actores implicados en el área de estudio que trata el proyecto. La Figura 2 representa el diseño de los ejes articuladores de los integrantes de La CAEC; son comportamientos que los caracterizan por su compromiso la experiencia, los conocimientos y decidir sobre los roles que se requieren para una determinada situación.

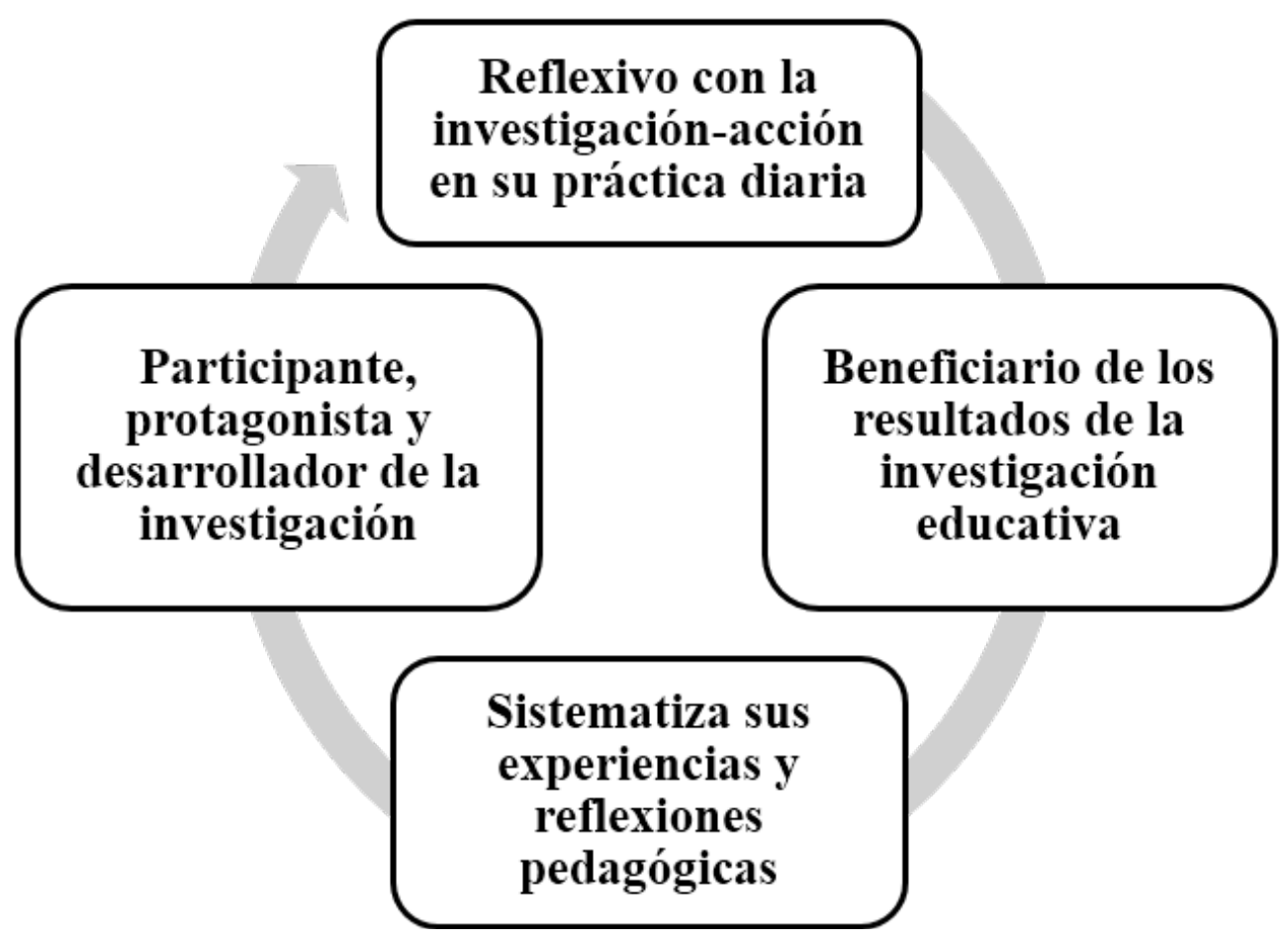

Figura 2. Diseño de ejes articuladores de La Comisión de Aprendizaje y Evaluación Continua. Fuente: Chaves y Barrios, 2015.

La CAEC se constituyó en una contribución para el mejoramiento continuo de las diferentes gestiones directivas, administrativas, pedagógicas y comunitarias, y se convirtió en una unidad integradora donde los agentes institucionales, la investigación y la práctica real, fueron elementos básicos en el proceso de la investigación-acción. Estos agentes se centraron en la observación del contexto y de sus propias prácticas pedagógicas de manera real y espontánea, y realizaron trabajo de campo en la medida que el maestro-investigador recogía información atendiendo a su doble rol: como sujeto observado y sujeto observador, para comprender y transformar la realidad. En este sentido, el trabajo de campo, la observación y el registro escrito de lo observado, fueron instrumentos que permitieron recoger información de la experiencia exploratoria, produciendo descripciones 
importantes para la investigación.

Los mencionados registros se originaron a partir de las prácticas educativas y de la contribución de los diferentes agentes integradores de la comunidad educativa, reconociéndole a cada miembro la capacidad de reunir y registrar libremente las manifestaciones intersubjetivas de los sujetos enfrentados a sus contextos reales, asumiendo actitudes de crítica y autocrítica que, en síntesis, condujeron a formular interrogantes de carácter teórico y conceptual con fines de transformación, innovación, reflexión y evaluación continua, producción intelectual y el diseño de propuestas para el mejoramiento de las gestiones institucionales. El uso de estas herramientas e instrumentos implicó el fortalecimiento de habilidades de observación, registro, análisis e interpretación. De igual forma, La CAEC planeó discusiones con los agentes educativos con el objetivo de profundizar en el significado de la experiencia colectiva del modelo pedagógico EpC, la construcción de proyectos pedagógicos y semilleros de investigación entre pares de distintas disciplinas.

Por otro lado, La CAEC diseñó las diferentes preguntas para formular en las entrevistas individuales a directivos, administrativos, maestros, estudiantes, padres de familia y a otros actores comprometidos en el área de estudio que trata el proyecto. Su objetivo era evaluar las impresiones subjetivas de los entrevistados en torno al desarrollo o actualización de competencias y su vinculación con su práctica cotidiana en el establecimiento y su disposición afectiva frente a su participación en proyectos interdisciplinarios y el modelo pedagógico EpC. Para ello, abordaba cuatro temas: evaluación y significado de su experiencia personal durante el proceso, evaluación de la experiencia de trabajo colectivo, aprendizajes obtenidos y proyecciones de esta experiencia educativa.

\section{Diseño metodológico}

El diseño metodológico de esta investigación toma como base el desarrollo pedagógico y educativo mediante un proceso de reflexión activo y participativo en el que intervienen los actores sociales involucrados en diferentes niveles de la institución educativa: directivos, administrativos, maestros, estudiantes, padres y miembros de la comunidad, con el objeto de lograr un mejoramiento institucional continuo de la práctica pedagógica para la generación de conocimiento y cambio social. En el esquema de la Figura 3 se sintetiza el diseño metodológico de este estudio, el cual inicia con el proceso de caracterización que pone en marcha un proceso reflexivo, el mismo que a su vez comienza por la interrogación de la realidad respecto de los problemas comunes que afectan a la comunidad educativa. Luego, viene la reflexión colectiva — más profunda - sobre los problemas comunes, para definir cómo y para qué intervenir de manera socialmente compartida. Seguidamente, se avanza hacia la sistematización de las experiencias, que consiste en una escritura reflexiva sobre las acciones que se realizan.

Finalmente, se asume la evaluación de las transformaciones alcanzadas para, a partir de ahí, avanzar hacia un nuevo ciclo de la investigación-acción en el que intervienen los actores sociales involucrados en la comunidad educativa.

En este caso, la calidad de la educación está unida a la investigación. Al contextualizar esta última, los agentes educativos reflexionan sobre su propias prácticas, de manera que al examinarla críticamente trasciende la misma crítica, la transforma, con el propósito de diseñar y aplicar nuevas estrategias y fases dirigidas a mejorar sus logros. Con ello, se da solución a los problemas, evaluación continua de teorías, transferencia metodológica, formulación de leyes o principios, metacognición y conocimiento.

Es importante resaltar que para la recolección de datos de los diferentes agentes educativos, se acudió a entrevistas, grupos de discusión, narraciones, diarios de campo, transcripciones de audio, video, y registros escritos de todo tipo, incluyendo lo observado. 


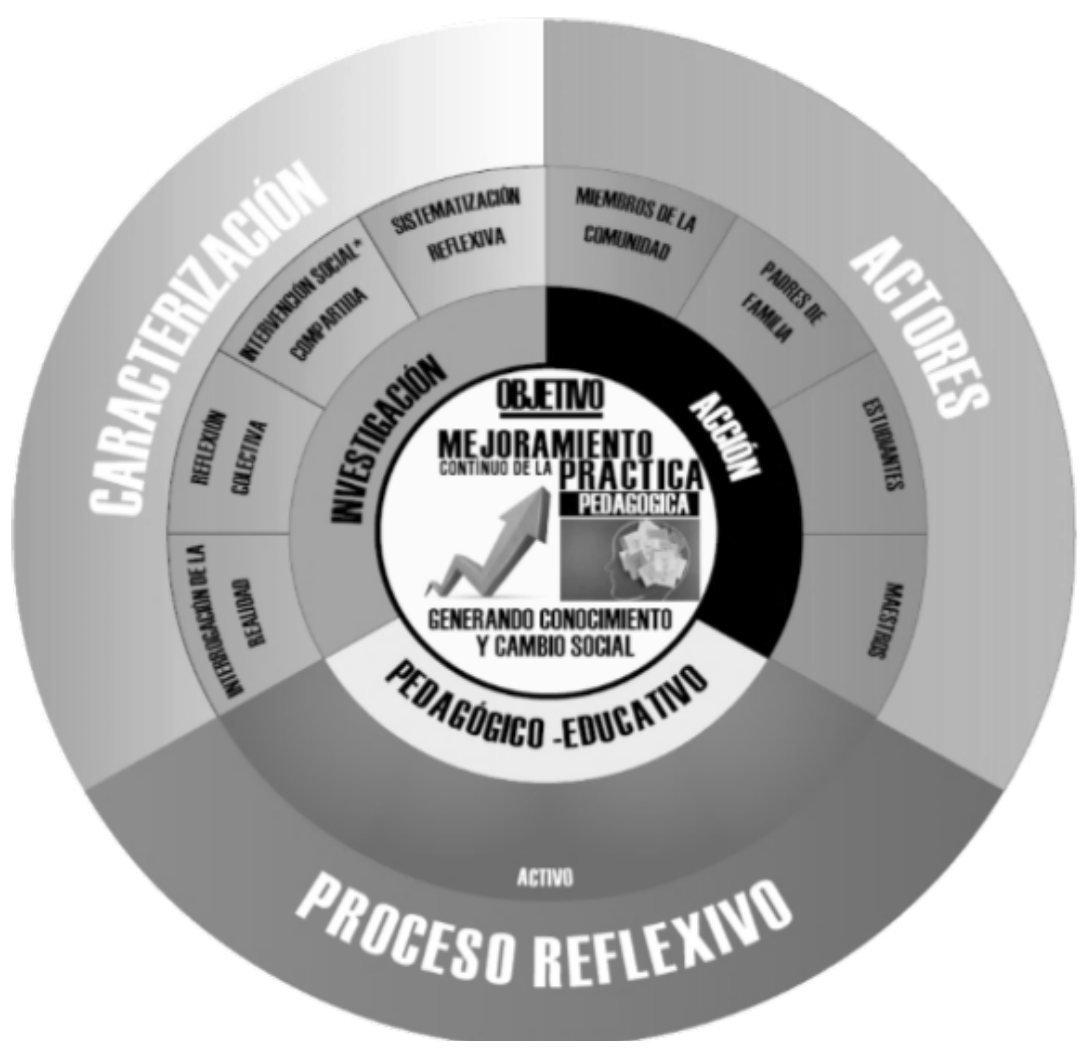

Figura 3. Esquema del diseño de la aplicación del método de investigación acción. Fuente: Chaves y Barrios, 2015.

Esta visión de la investigación-acción en los procesos escolares propone un modelo cíclico de reflexión-acción-reflexión en el que se reorganiza la relación entre conocer y hacer, entre sujeto y objeto, configurando y consolidando con cada paso la capacidad de autogestión de los implicados. Así, se configura un contexto investigativo más abierto y procesual (Bru y Basagoiti, 2002).

Para el análisis de la investigación desde el enfoque cualitativo, se utilizó la herramienta computacional ATLAS.ti. El procedimiento de la técnica de análisis se realizó a partir de los diferentes datos e informes recolectados que las investigadoras codificaron y posteriormente analizaron de forma simultánea para desarrollar conceptos. De igual forma, se siguieron unas reglas de sintaxis que establece el programa y los parámetros que establecieron las investigadoras.

Con la ayuda del ATLAS.ti se diseñó una matriz de análisis con las categorías, rasgos, descriptores, microtextos de las entrevistas y comentarios, para la relación, comprensión, comparación y contrastación de los hallazgos. Desde las interpretaciones realizadas, la estructuración de hallazgos y la teorización, son creaciones libres que surgieron de la realidad estudiada y de la creatividad del grupo de trabajo conformado por La CAEC y las investigadoras.

En este sentido, Kaplan (1964) señala que solo se llega a una buena teoría mediante el ejercicio de la imaginación creativa, ya que la formación de una teoría no consiste en descubrir o en destapar un hecho escondido, sino que es un modo de mirar los hechos, un modo de organizarlos y representarlos conceptualmente a través de una nueva red de relaciones entre sus partes constituyentes. Aunque la teoría se ajusta y acomoda en cierto modo al mundo exterior, en otro sentido importante crea un mundo propio. 


\section{Resultados}

En ésta sección se presentan los resultados del presente estudio. En tal sentido, Salcedo (2001) anota:

La investigación educativa como posibilitadora de construcción de conocimiento se convierte en instrumento para descubrir el mundo complejo de la escuela. La investigación como base de la enseñanza y de formación, permite al docente desde la reflexión, la construcción de saber dejando de lado el rol de mediador pasivo entre teoría y práctica, sin embargo, como bien lo ha señalado Porlán (1995) solo una reflexión que incorpore la crítica ideológica (Carr y Kemmis, 1988:168) puede revelar a los educadores como sus creencias y actitudes quizás sean ilusiones ideológicas que ayudan a preservar un orden social ajeno a sus experiencias y necesidades colectivas. El ejercicio investigativo puede dirigir procesos reales de transformación no solo de las prácticas pedagógicas sino de las prácticas sociales inmersas en la Escuela, favoreciendo la democratización del conocimiento y atenuando los efectos de la desigualdad social. (párr. 5)

De esta manera, las investigadoras demuestran que la investigación sirvió para implementar e intensificar la reflexión-acción-reflexión en el equipo de docentes partiendo de presupuestos particulares, para luego ser escuchados por el equipo de investigación La CAEC, logrando un acercamiento entre el pensamiento reflexivo del docente y su práctica pedagógica.

Como puede apreciarse en la gráfica de los resultados de la investigación, inserta debajo, los maestros identificaron la importancia de integrarle a la práctica educativa la reflexión con la investigación acción en su práctica diaria, tendencia que les permitió ser participantes y protagonistas en el desarrollo de la investigación; además, favoreció entre el colectivo de docentes la conformación del equipo de investigación La CAEC, mejorando las prácticas y estrategias pedagógicas en el aula. En este sentido, afirmamos que las competencias y habilidades pedagógicas del maestro inciden de manera directa en el aprendizaje de los estudiantes.

\section{DINAMIZACIÓN DE LOS PROCESOS ENSEÑANZA Y APRENDIZAJE}

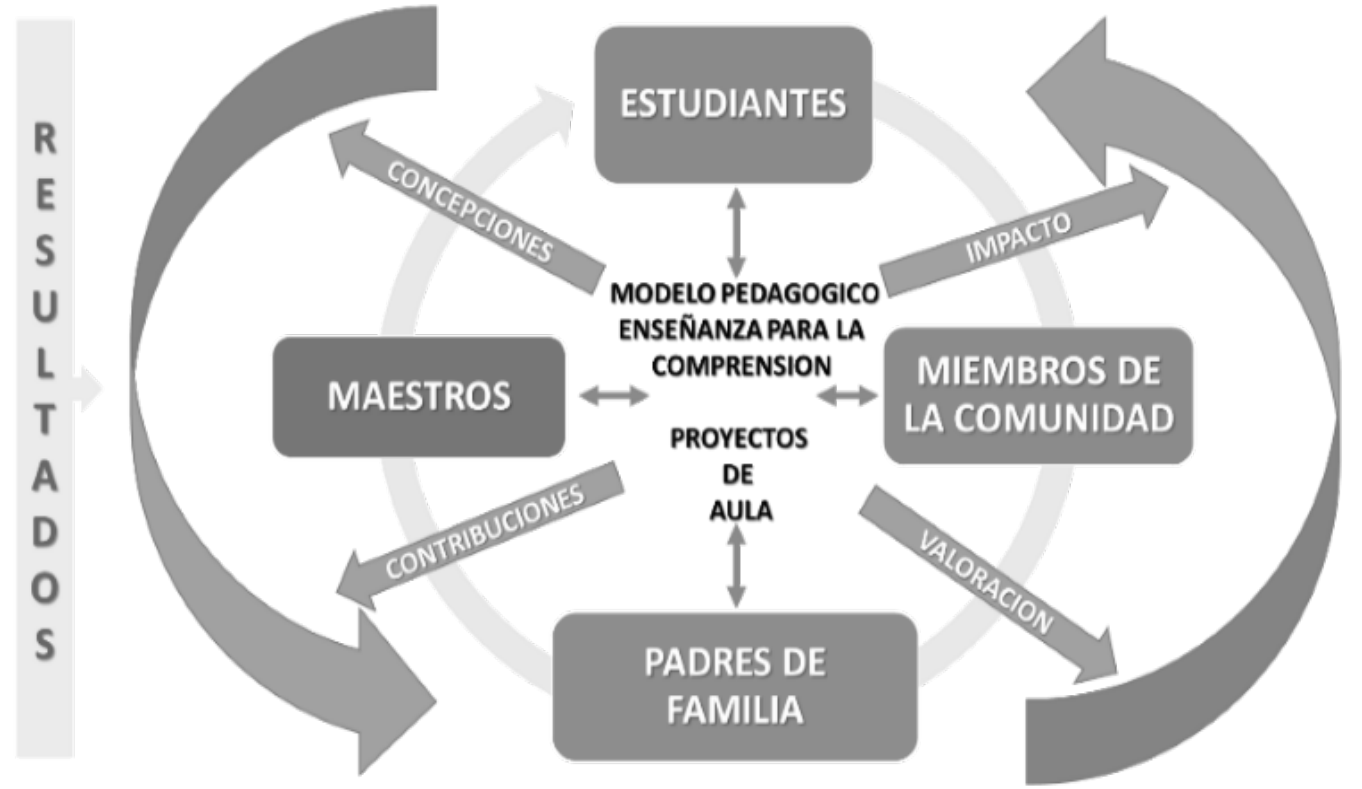

Figura 4. Esquema de dinamización de los procesos de enseñanza y aprendizaje. Fuente: Chaves y Barrios, 2015. 
Por otro lado, la implementación del proyecto de aula en el marco del modelo pedagógico EpC, como estrategia didáctica y de formulación, planeación, ejecución y sistematización del mismo, impacta no solo en los estudiantes sino también en las familias, docentes y comunidad educativa. Podemos decir, que es una propuesta de cambio y transformación para el desarrollo de competencias convivenciales y ciudadanas.

Las voces de los docentes en los resultados de la investigación permitieron evidenciar el mejoramiento continuo de los procesos de enseñanza, identificando en el estudiante los conocimientos previos y las interacciones entre docente-estudiante-familia-contexto, escuchando y atendiendo las dificultades de sus estudiantes.

Al mismo tiempo, con la orientación de los docentes, los estudiantes pueden identificar puntos fuertes y débiles en el desarrollo de sus competencias para la vida y participar así de los procesos de mejoramiento continuo y de evaluación en el aula. En este sentido, los proyectos de aula fomentan los canales de comunicación entre los distintos miembros de la comunidad educativa (docentes, directivos, estudiantes, familias) y hacen que se compartan metas educativas comunes, relacionadas con una formación integral y con la adquisición de las competencias necesarias para desempeñarse efectivamente en la sociedad.

Por su parte, los padres de familia se informan sobre la calidad de la educación que reciben sus hijos, y así, analizan y contribuyen al mejoramiento de su desempeño y al de la misma institución frente a los ideales esperados; igualmente, obtienen argumentos y criterios para interactuar con las distintas actividades organizadas por los docentes en torno a la formación de los estudiantes.

De esta manera, se indica que los procesos académicos parten del modelo pedagógico EpC, evidenciando que el modelo mira hacia el estudiante, lo reconoce como un sujeto con voz, con conciencia y sobre todo, con inteligencia. En este viraje, el sentido de la formación tiene que ver con el desarrollo de habilidades y competencias en el escolar, como una apuesta de reconstrucción de lo humano en el marco de la complejidad de lo social.

Después de ejecutar el proyecto, indicamos que la investigación-acción no se constituye solo en un ejercicio individual, sino que está inmersa en las complejas interacciones entre el docente, los estudiantes, los padres de familia, el contexto social y las relaciones entre pares que se desarrollan en los entornos educativos y, sobre todo, resulta fundamental al enfrentarnos con los problemas de la calidad educativa, los cambios intencionales de las prácticas escolares, y el impacto y la transformación del mismo contexto social.

Resulta asimismo interesante comprobar que una educación de calidad para población en condiciones de pobreza no solo es necesaria sino posible, pues reduce la fuerte estratificación que persiste en el sistema educativo local. Todo ello a pesar de que los recursos económicos que el Estado asigna a esas instituciones son iguales o inferiores a los que asigna a cualquier otra institución educativa pública.

\section{Discusión}

Los resultados señalan cambios significativos en las prácticas docentes luego de implementar los proyectos de aula. En este sentido, Schon (1987), manifiesta que para el docente, el proyecto de aula significa «descubrir cómo nuestro conocimiento en la acción pudo haber contribuido a ese resultado inesperado» (p. 27). Mientras que para Sayago (2003), los docentes deben tener previamente el conocimiento integrador del proyecto de aula, con orientaciones de contenidos generales de las diferentes áreas, para el desarrollo de competencias en los estudiantes.

Si bien los docentes destacan los cambios observados en los estudiantes producto de la experiencia de 
los proyectos de aula, algunos encuentran dificultades en el desarrollo de sus proyectos por cuenta de la falta de disponibilidad horaria y por el débil apoyo de los entes administrativos de la institución educativa.

No obstante, algunos docentes señalaron sentirse muy motivados para seguir trabajando en proyectos de aula, pero a la vez preocupados por los costos de los materiales que el colegio no les facilitaba y que en la mayoría de los casos son suministrados ellos mismos.

En este sentido, Sayago (2003), dentro de una lectura dialógica-crítica, presenta los proyectos de aula como consecuencia de los Proyectos Institucionales, procurando así la coherencia entre los objetivos pedagógicos del establecimiento educativo y la transformación de las prácticas docentes, el aprendizaje de los estudiantes y el impacto social. Este aporte teórico debe ser evidente en las instituciones educativas que trabajan bajo el enfoque de la enseñanza por proyectos, ya que desde allí se permean todos los escenarios de la vida académica y convivencial de la escuela bajo impactos realmente sostenibles a largo plazo.

El diario de campo, la observación y la sistematización de las experiencias se establecieron como instrumentos de recolección de información básicos, que gracias al acompañamiento oportuno, paulatinamente se fueron adoptando en el proceso investigativo. Su impacto en el proceso de reflexiónacción fue tan importante, que los implicados en el proceso investigativo se sintieron como ejes centrales de esta experiencia de aprendizaje y trabajo colaborativo.

En el proceso de reflexión-acción-reflexión colectiva, la investigación-acción se vio acompañada con expresiones de sentimiento frente a la situación de vulnerabilidad de los estudiantes y sus familias, siendo a la vez eje motivacional e impulsador para que a través de los procesos de calidad educativa los estudiantes logren posteriormente el ingreso a la educación superior e impacten positivamente sobre el desarrollo social de su contexto. En este sentido, es una competencia que un profesional muestra en situaciones de la práctica que resultan singulares, inciertas y conflictivas, cuando ha llevado a cabo procesos de reflexión en la acción (Schön, 1987).

Así pues, el proceso de reflexión-acción-reflexión es continuo; por lo tanto, se hace necesario que el grupo investigador, La CAEC y las directivas del colegio, mantengan como fundamento institucional la investigación y la calidad educativa desde las aulas.

Es interesante observar cómo los profesores progresivamente van incorporando nuevas prácticas educativas en su quehacer pedagógico en un proceso de resignificación evaluativo y de mejoramiento continuo que se traduce en un impacto positivo sobre la comunidad educativa. Al resaltar la práctica reflexiva planteada por los maestros de la institución educativa, se presentan situaciones similares a las sugeridas por Schön (1987), cuando menciona que el desarrollo de las competencias eficaces del docente y despiertan en el estudiante un pensamiento crítico y participativo, siendo este último el directamente beneficiado de su proceso de aprendizaje, pues le permite impactar en el desarrollo de su propio contexto.

En este orden de ideas, para mantener y enriquecer la calidad educativa y la transformación y el desarrollo social, es necesario incrementar el apoyo a la actividad de investigación educativa. De igual forma, se requieren esfuerzos organizacionales permanentes y sistemáticos con el fin de apoyar la erradicación del trabajo infantil, el maltrato infantil, el reclutamiento forzoso, entre otras dificultades que atraviesan los estudiantes de la institución. 


\section{Conclusiones}

En esta investigación se evidencia el impacto en la calidad educativa del modelo pedagógico Enseñanza para la Comprensión (EpC) y los proyectos de aula, además de la trascendencia de las prácticas docentes en relación directa con la formación de los maestros, los logros de los estudiantes y la incidencia de las familias. Sin embargo, también se evidencia que tales prácticas están determinadas por el contexto social, aun cuando su desarrollo y evolución son cotidianos, dado que se renuevan y originan durante las sesiones de clase, brindando soluciones espontáneas ante diversas situaciones. De igual forma, la apropiación que cada maestro hace le permite formarse de manera continua, actualizar sus conocimientos y asumir compromisos influenciados por escenarios, dentro y fuera del aula y la escuela.

En este sentido, las reflexiones propuestas en esta investigación permiten reconocer la importancia de los proyectos de aula en la práctica educativa, circunstancia que consolida la innovación en el aula y la construcción de alternativas que propician aprendizajes pertinentes a partir del análisis del contexto en que viven los estudiantes, a través de la indagación e identificación de problemas, emprendiendo acciones de desarrollo humano sostenible. De igual forma, se evidencia que el colegio ha incentivado y permitido la integración de los padres de familia y acudientes en la acción educativa. De esta forma, se visibiliza con mayor preponderancia la participación de los padres de familia en el proceso de enseñanza y aprendizaje, ratificando que la familia es el ámbito natural del desarrollo de los niños.

Asimismo, a los estudiantes les gusta aprender de forma compleja y dinámica, demostrando que el complemento a la planeación amplía las perspectivas para tratar los temas según las estrategias didácticas utilizadas. A los estudiantes les impacta y motiva la diversificación de estrategias de enseñanza para aprender activamente, y cuando encuentran estos elementos en el aula, los valoran como un aporte de los docentes a procesos de aprendizaje que los incluyen en forma significativa. De la misma forma, los estudiantes ven que los proyectos de aula son significativos para lo que viven fuera de la escuela, dado que consolidan los saberes, las tareas responden a sus intereses, las evaluaciones contribuyen a reforzar sus conocimientos y, en este sentido, encuentran que la educación tendrá un significado importante, tanto para ellos como para sus familias.

Se indica asimismo, la necesidad de consolidar una educación pertinente y de calidad en la escuela. Aquí es donde se establece un ineludible compromiso ético con el desarrollo de competencias —en términos de lo que saben y son capaces de hacer- que concedan a los estudiantes la capacidad de desarrollar habilidades, conocimientos y valores que les permitan comprender, producir y transformar conocimiento e interactuar con el mundo en el que viven.

Finalmente, el modelo pedagógico Enseñanza para la Comprensión (EpC) y la enseñanza por proyectos se constituyen en insumos para la construcción de planes de mejoramiento, la construcción, deconstrucción y reconstrucción de los currículos y el cambio en las prácticas pedagógicas con el fin de fortalecer las prácticas de aula. De igual forma, los resultados de este proyecto de investigación presentan insumos para el mejoramiento continuo, la vinculación de la enseñanza y la investigación, mantener y fortalecer la calidad educativa en la escuela; además, también aporta instrumentos para saber qué es lo que sucede en el aula, cómo aprenden los alumnos, cómo se puede organizar para ello el espacio y el tiempo, y qué estrategias de intervención pueden ser las más oportunas en determinado contexto.

\section{Referencias}

Bru M, P., \& Basagoiti, M. (2002). La investigación-acción participativa como metodología de mediación e integración socio-comunitaria. Disponible en: www.pacap.net/es/publicaciones/ pdf/comunidad/6/documentos_investigacion.pdp

Carr, W., \& Kemmis, S. (1986). Teoría crítica de la enseñanza. Barcelona: Martínez. 
El proyecto de aula como estrategia didáctica

Chaves, M., \& Barrios, L. (2015). El proyecto de aula como estrategia didáctica en el marco del modelo pedagógico enseñanza para la comprensión. (Tesis de Maestría). Universidad de Córdoba, Montería, Colombia.

Coll, C. (1981). Algunos problemas planteados por la metodología observacional: Niveles de descripción e instrumentos de validación. Anuario de Psicología, 24 (I), pp. 111-131.

Coll, C. \& Valls, E. (1993). El aprendizaje y la enseñanza de los procedimientos en los contenidos de la reforma. Barcelona: Morata.

Denegri, M. (1996). Las ideas de Piaget y Vigotsky. Santiago de Chile: La Salle.

Denegri, M., \& Martínez, G. (2001). Proyectos de aula interdisciplinarios: una mirada desde la psicología educacional. En Psicología y Educación: Encuentros y desencuentros. María Juliá y Jorge Catalán (Eds.) Departamento de Publicaciones Universidad de La Serena.

Doria C, R. (noviembre, 2011). El currículo: eje de la gestión escolar para la formación y el desarrollo humano. Universidad de Córdoba, Colombia.

Gimeno, S. \& Pérez G, A. (1996). Comprender y transformar la enseñanza. Madrid: Morata.

Guzmán, B., Graciela, S., \& Jiménez, P. (1991). «El aula: espacio de interrelación de quehaceres y finalidades educativas». En: El aula universitaria, México: UNAM.

Kaplan, A. (1964). The conduct of inquiry: methodology for behavioral Science. San Francisco, CA: Chandler Publishing Co.

Kemmis, S. (1992). El currículum: va más allá de la teoría de la reproducción. Madrid: Morata.

Kemmis, S., \& McTaggart, R. (1992). Cómo planificar la investigación-acción. Barcelona: Laertes.

Le Compte, M. D., Millroy, W. L. \& Preissle, J. (Eds.). (1995). The handbook of qualitative research in education. California: Academic Press.

Lewin, K. (1948). La resolución de los conflictos sociales; trabajos seleccionados en la dinámica de grupo. Gertrude W. Lewin (ed.). Nueva York: Harper \& Row.

Marcelo, C. (2001). Aprender a enseñar para la Sociedad del Conocimiento. Revista Complutense de Educación. Vol. (12) 2, pp. 531-593.

Negrete, V., \& Garcés, M. (2010). Análisis sociopolítico de Montería y propuestas sobre liderazgo, participación y compromiso ciudadano. Revista Universidad del Sinú. Montería.

Not, L. (1992). La Enseñanza Dialogante. México: Editorial Herder.

Pérez Serrano, G. (1998). Investigación cualitativa. Retos e interrogantes. I. Métodos. Madrid: La Muralla.

Perkins, D. (1999). La Escuela Inteligente. Del adiestramiento de la memoria a la educación de la mente. Barcelona: Gedisa.

Pogré, P. (2001). Enseñanza para la Comprensión. Un marco para innovar en la intervención didáctica. Buenos Aires: Papers.

Rodríguez, S. (2000). El Proyecto de aula como estrategia pedagógica. (Proyecto de grado). Universidad de la Sabana, Chía, Colombia.

Sandín E, M. P. (2003) Investigación Cualitativa en Educación. Fundamentos y Tradiciones. Madrid: McGraw-Hill.

Sayago, Z. (2003). Los proyectos pedagógicos de aula: Entre lo real y lo posible. Revista Educere Trasvase, 23, pp. 421-422.

Schön, D. (1987). El profesional reflexivo. Cómo piensan los profesionales cuando actúan. Barcelona: Paidós.

Siemens, G. (2004). Conectivismo: Una teoría de aprendizaje para la era digital. Disponible en: http:// www.diegoleal.org/docs/2007/Siemens 
Stenhouse, L. (1984). La investigación como base de la enseñanza. Madrid: Morata.

Stone W, M. (1999). Enseñanza para la comprensión. Buenos Aires: Paidós.

Unesco (2013). Hacia la educación de calidad para todos al 2015. Santiago de Chile, Chile.

Vázquez, R. (2011). Enseñanza para la comprensión: El caso de la escuela rural de Bolonia (Cádiz, España). Revista Iberoamericana de Educación, 57, pp. 183-202.

Wiske, M. (1999). La enseñanza para la comprensión. Vinculación entre la investigación y la práctica. Buenos Aires: Paidós.

Zabala V, A. (1995). La práctica educativa: Cómo enseñar. Barcelona: Graó. 